\title{
In-silico design of novel myocilin inhibitors for glaucoma therapy
}

\author{
Min Tang, Yang Fu, Ying Fan, Ming-Shui Fu, Zhi Zheng and Xun Xu* \\ Department of Ophthalmology, Shanghai general Hospital of Nanjing Medical University, Shanghai 200080, China
}

${ }^{*}$ For correspondence: Email: xuxun9012@hotmail.com; Tel: 0086-021-63240090; Fax: 0086-021-36126336

Sent for review: 6 June 2016

Revised accepted: 22 September 2017

\begin{abstract}
Purpose: To explore newer computational approaches in the design of novel myocilin inhibitors for the treatment of glaucoma.

Methods: An in-silico virtual screening technique based on simulation of molecular docking was utilised to design a novel myocilin inhibitors for the treatment of glaucoma. The designed novel molecules were theoretically evaluated to predict their pharmacokinetic properties and toxic effects. Lead molecules were screened out in virtual screening technique on the basis of low binding energies obtained in AutoDock based molecular docking simulation.

Results: Out of ten top lead compounds screened, ZINC01729523 and ZINC04692015 were promising, having shown potent inhibition of myocilin, good pharmacokinetic properties and absence of any toxic effects.

Conclusion: In-silico virtual screening of molecular libraries containing a large number of ligands is very useful for short-listing of potential lead molecules for further structure-based discovery of antiglaucoma-drugs.
\end{abstract}

Keywords: Glaucoma, Myocilin, Docking, Virtual-screening, Autodock, Ligand, Drug design

Tropical Journal of Pharmaceutical Research is indexed by Science Citation Index (SciSearch), Scopus, International Pharmaceutical Abstract, Chemical Abstracts, Embase, Index Copernicus, EBSCO, African Index Medicus, JournalSeek, Journal Citation Reports/Science Edition, Directory of Open Access Journals (DOAJ), African Journal Online, Bioline International, Open-J-Gate and Pharmacy Abstracts

\section{INTRODUCTION}

Glaucoma is the loss of visual sensitivity and field because of optic neuropathy associated with damage to the optic nerve heads. Traditionally, glaucoma is diagnosed by increased intra-ocular pressure (IOP), which is a very crucial factor in its pathogenesis. The normal range of IOP in healthy individuals is 10 to $21 \mathrm{mmHg}$ [1-3]. An increase in IOP above this range creates a pressure against the optic nerve, resulting in the death of the nerve cells. Increased IOP leads to improper draining of the aqueous humour produced by the chambers of the eye between the cornea and the lens [1-3]. Glaucoma is associated with mutations in myocilin. Studies have shown that alterations in the size, electrical charge and polarity of myocilin due changes in its peptide sequence are important for development of glaucoma [4-7].

According to the vasogenic theory, factors such as insufficient blood flow to retina due to increased perfusion pressure required in the eye, dysregulated perfusion, and vessel wall abnormalities damage the optic nerve by degeneration of axonal fibres of the retina [5-8]. Another theory suggests that the IOP may disrupt axoplasmic flow at the optic disk. Myocilin is produced by most ocular tissues such as iris, sclera, lens, cornea, retina ciliary body, optic nerves and trabecular meshwork (TM) [5-8]. Myocilin is present in the intracellular and extracellular portions of tissues. It is expressed in 
TM cells which are responsible for the generation of resistance to out flow of aqueous humour; this resistance is accounts for the elevation of IOP and glaucoma [5-8]. World Health Organization (WHO) has reported that glaucoma is the second leading cause of blindness worldwide, and that about 70 million people across the age groups are affected by the disease. Multiple populationbased surveys estimated that about 79.6 million individuals would be affected by glaucoma by the year 2020 [8-10]. The increasing incidence of glaucoma across the world makes it expedient to develop a potent drug for its treatment.

In the present study, in-silico approaches were applied to identify some myocilin inhibitors as potential sources of novel anti-glaucoma drugs.

\section{EXPERIMENTAL}

All the molecular docking simulations were carried out utilizing Raccoon, AutoGrid4 and AutoDock4 software [11].

\section{Selection and preparation of protein}

Human Myocilin Olfactomedin Domain protein bound to its endogenous ligand hexaethylene glycol (pdb id-4WXQ) was downloaded from RCSB protein data bank. The $4 \mathrm{WXQ}$ protein complex consists of a single polypeptide chain of 277 amino acids [12]. The receptor protein myocilin was prepared for molecular docking by removing ligand and from active site, removal of water molecules which are not interacting with the ligand, and addition of polar hydrogen's. The amino acid residues GLY252, LYS468, GLU253, TRP250, VAL251 and ARG470 are present in the active site of protein $4 \mathrm{WXQ}$ [12].

\section{Selection of chemical libraries}

Diverse molecules (1880) present in the $\mathrm{NCl}$ "Diversity Set-II" molecular library were virtually screened to identify possible lead compounds. All the ligands used in virtual screening followed Lipinsky's rule of five $(\mathrm{ClogP}<5 ; \mathrm{H}$ bond donor < 5; $\mathrm{H}$ bond acceptor < 10; molecular weight $<500$ ) for good pharmacokinetic properties [13-14].

\section{Grid box formation}

Table 1 shows the grid box points of the three proteins. These were utilized for all docking runs. The grid box was placed in the center of the ligand to ensure that all the extended conformations of ligand fit into the grid box.

\section{Preparation of grid map}

The map files for different atom types in ligands and receptor viz. A, OA,C, HD, F, I, Br, Cl, N, NA, SA, $S$ etc. were prepared by running Autogrid utility of the AutoDock suite. These map files were prepared by Autogrid and used by AutoDock for carrying out molecular docking simulations.

\section{Docking parameters}

Lamarckian genetic algorithm (LGA) is the primary conformational search approach in AutoDock [11]. In this search, a trail population is created for various possible conformations, and mutations and exchange of conformational parameters take place to compete in a similar manner to that of the successive generations for biological evolution by the selection of the final individuals with minimum binding energy. The "Lamarckian" aspect is used for finding the local minima, and the local conformational space of the individual conformational search. These information's are passed to later generations. Semi-empirical free energy force field is used to predict binding free energies of small molecules to macromolecular targets. The force field is based on a comprehensive thermodynamic model-based force field that allows incorporation of intra-molecular energies into the predicted binding free energy by evaluating energies for both the bound and unbound states. Docking parameter file was prepared for each ligand using the following conditions: number of GA runs was 150; maximum number of evaluations (short) was 250000; maximum number of generations was 27000; number of GA runs was 10 , and the rate of gene mutation was 0.02 $[13,14]$.

\section{Validation of docking method}

The positions and orientations of the ligand obtained after the molecular docking study represented potential binding modes of the inhibitors. The various docking parameters considered in the docking methods were validated by re-docking individually crystallized hexaethyleneglycol (ligand) over Myocilin Olfactomedin Domain protein of Homo-sapiens.

Table 1: Coordinates of grid box for the three proteins

\begin{tabular}{cccccccc}
\hline Proteins & x-D & y-D & z-D & Spacing $(\hat{\mathbf{A}})$ & x center & y center & z center \\
$4 \mathrm{WXQ}$ & 40 & 40 & 40 & 0.381 & -2.133 & -26.467 & -21.434 \\
\hline
\end{tabular}


The docked conformation of the bound ligand must perfectly overlap the crystallized chemical structure to obtain root mean square deviation (RMSD) values within acceptable range [11-14].

\section{Virtual screening process}

The files necessary for performing virtual screening process were prepared by using the software Raccoon. Raccoon is a graphical user interface for AutoDock-based virtual screening of ligand libraries against a macromolecule. It is used to split molecular ligand library file having multiple number of ligands, to convert them into the pdbqt format required by AutoDock, and filter the unwanted ligands by using some common criteria, such as Lipinski's rules, fragment-like "rule of 3" and drug-likeness. Input file is validated at every step by evaluating the presence of non-standard atom types and ensuring that parameters, input filenames, and grid maps have coherent formats $[13,14]$.

\section{Analysis of results of docking simulation}

A script Summarize_results.py from Scripps Research Institute was utilized to sort the binding energies of the docked ligand and select the best hits. The results obtained from molecular docking simulation were evaluated on the basis of hydrophobic and polar interactions obtained between ligand and the binding residues present in the active ligand binding site of the macromolecule. The empirical range of the binding free energy should be in the range of -5 to $-15 \mathrm{Kcal} / \mathrm{Mol}$. Binding affinities were calculated as in Eq 1.

$K_{\mathrm{i}}=\mathrm{e}^{[(\Delta \mathrm{G} /(\mathrm{RT})]}$

where $\Delta G=$ change in free energy upon binding, $R=$ gas constant and $T=$ absolute temperature [13].

\section{Prediction of absorption, distribution, metabolism, excretion (ADME) and toxicity of lead compounds}

The toxic effects of the virtually-screened lead molecules were predicted by using the OSIRIS online program. The presence of major toxic effects such as mutagenicity, tumorigenicity, irritant effects and reproductive effects were predicted on the basis of functional groups present in the chemical structures of the lead compounds. This program also calculates druglikeness score and drug score of the lead molecules on the basis of their physicochemical properties [13-15].

\section{RESULTS}

Table 2 depicts the results of the internal validation for the proteins $1 \mathrm{AM} 4$ showing that the root mean square deviation (RMSD) value obtained conformed with the acceptable value of less than $1 \AA$. This indicates that the docked conformation of the ligand has similar binding mode to that present in its bioactive conformation. In addition, a very good binding affinity value was obtained in internal validation of the molecular docking process. The binding residues involved in the binding of the ligand to the macromolecule are shown in Table 2.

Figure 1 illustrates the binding mode and chemical interactions of the ligand hexa-ethylene glycol within the active binding site of human Myocilin Olfactomedin Domain protein, while Figure-2 shows the overlay of chemical structures of docked conformation of the ligand with respect to its crystallized bioactive conformation. Both the figures indicate that the docked conformation of the ligand has exactly the same binding mode and chemical interactions with the macromolecule as the bioactive conformation of the ligand. These results clearly suggest that the ligand binding mode obtained by molecular docking technique simulated exactly the binding mode observed within the human body.

The potential ligands were selected by analysing the ligand-protein interactions for top-ranking pose of each ligand, and interactions of docked compound were visualized. Virtual screening results for human myocilin protein are enumerated in Table 3. Table 4 illustrates that all ten hits followed Lipinski's rule of five [13-15].

The ligand-protein interactions in 4WQX were compared with the interactions of the shortlisted lead molecules. The best poses were identified using the following criteria in the given order of preference: lowest binding energy in the largestsized cluster, number of hydrogen bonds with active site residues and conservation of interactions with those from control docking. This was performed to ensure that the hits were actually docking exactly into the right binding site of interest.

Two of the top ten screened lead molecules (ZINC01729523 and ZINC04692015) showed promising results on the bases of potent inhibition of myocilin, good pharmacokinetic properties, absence of toxic effects, as well as very good drug-likeness and acceptable Drug Score values. The ADME and toxicity results of 
all the lead molecules obtained by virtual screening are shown in Figure 3. Some of the designed inhibitors showed poor pharmacokinetic profiles, high toxicity, mutagenicity, tumorogenicity and reproductive effects.

Table 2: Internal validation results for the three proteins

\begin{tabular}{ccccc}
\hline Protein & Interacting residue & $\begin{array}{c}\text { Internal } \\
\text { validation } \\
\text { RMSD value }\end{array}$ & $\begin{array}{c}\text { Binding energy } \\
\text { (Kcal/Mol) }\end{array}$ & $\begin{array}{c}\text { Binding affinity } \\
\text { (nM) }\end{array}$ \\
\hline 4WXQ & GLY252, GLU253, & 0.84 & -5.29 & 132.17 \\
& TRP250 and VAL251 & & & \\
\hline
\end{tabular}

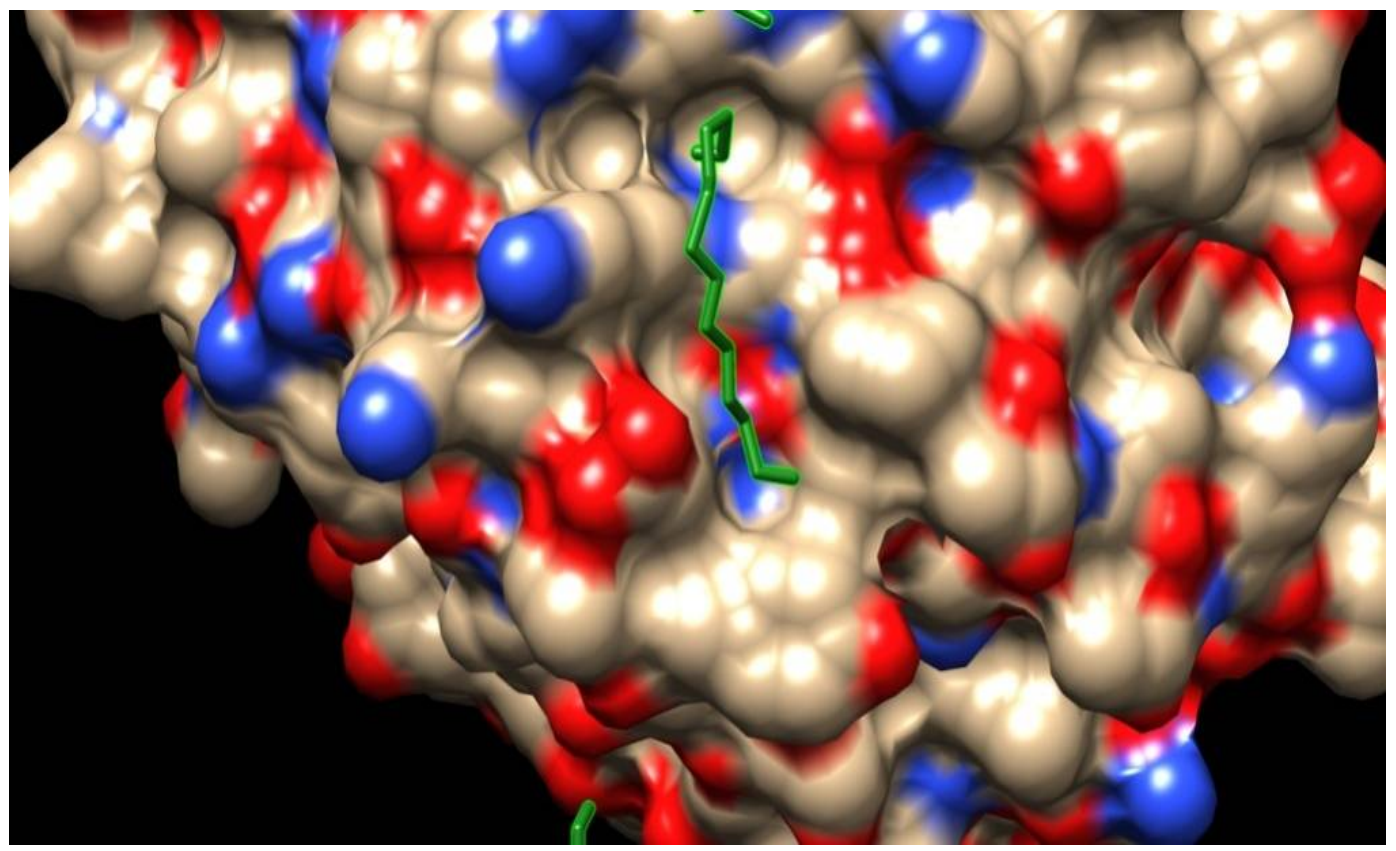

Figure 1: Binding mode and chemical interactions of the ligand hexaethylene glycol within the active binding site of human Myocilin Olfactomedin Domain protein

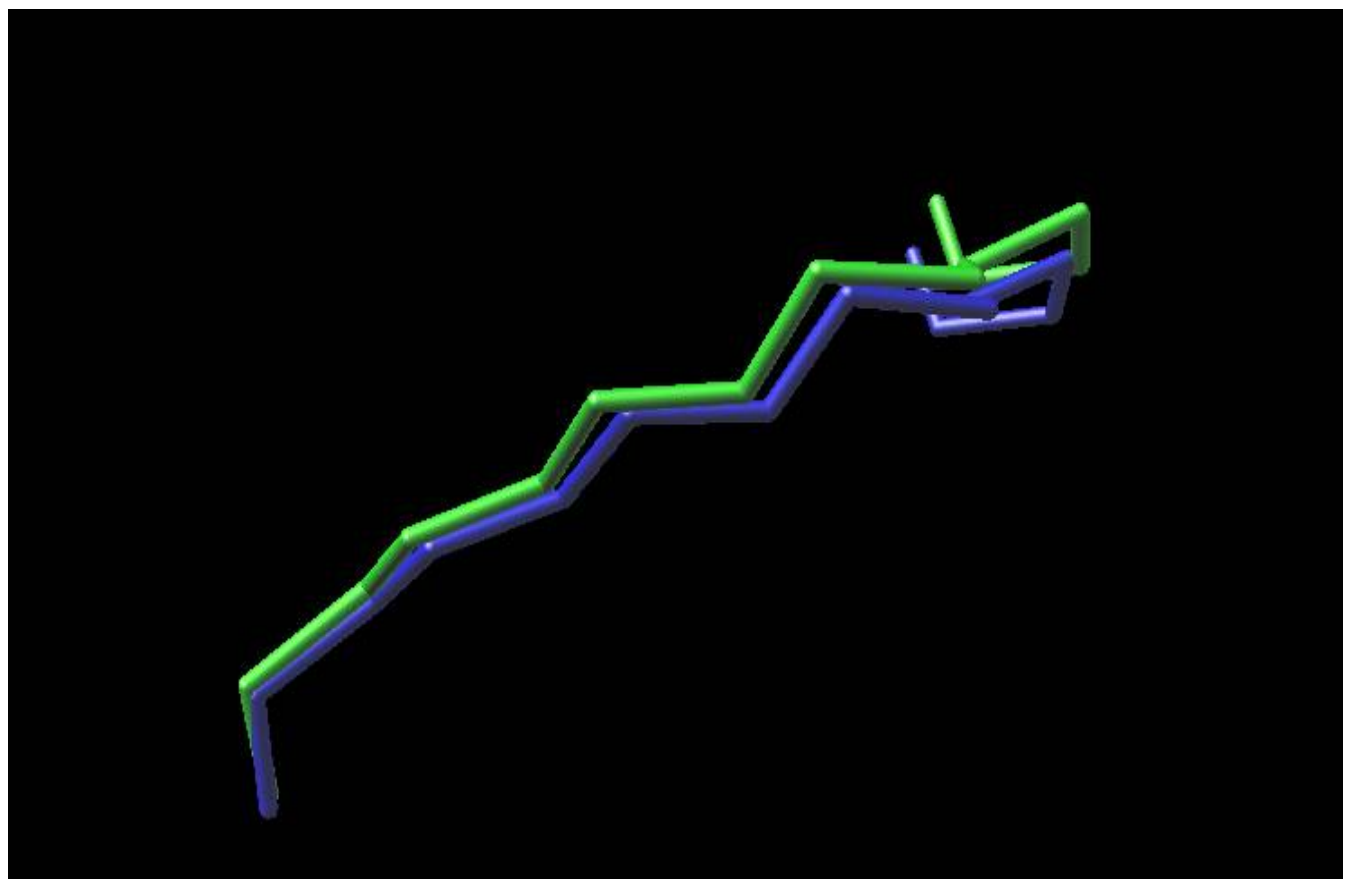

Figure 2: Overlay of chemical structures of docked conformation of the ligand with respect to its crystallized bioactive conformation [12] 
Table 3: Binding energies and affinities of five top hits from $\mathrm{NCl}$ "Diversity Set II" after virtual screening on Human Myocilin Olfactomedin domain protein

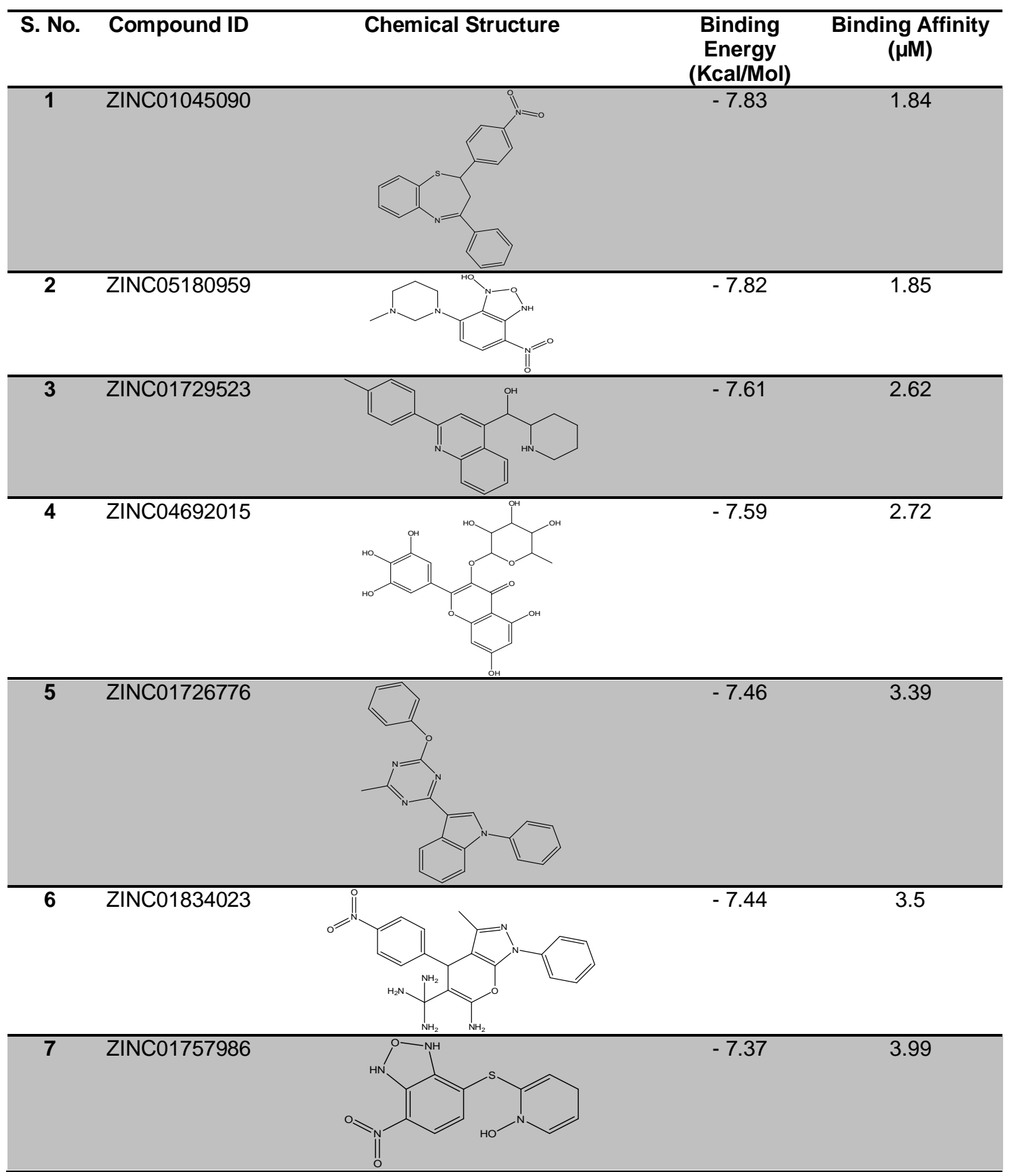

Table 4: "Lipinski's rule of five" for the hits on human myocilin protein

\begin{tabular}{lccccc}
\hline Compound ID & Mol. Wt. & Clog $\boldsymbol{P}$ & 2D PSA $\left(\dot{A}^{2}\right)$ & HBA & HBD \\
\hline ZINC01045090 & 374.45 & 6.06 & 58.18 & 5 & 0 \\
\hline ZINC05180959 & 281.20 & -0.18 & 97.03 & 10 & 2 \\
\hline ZINC01729523 & 332.43 & 4.52 & 45.15 & 3 & 2 \\
\hline ZINC04692015 & 464.37 & -0.94 & 206.60 & 13 & 8 \\
\hline ZINC01726776 & 348.42 & 7.06 & 52.83 & 3 & 0 \\
\hline ZINC01834023 & 407.42 & 1.76 & 176.95 & 10 & 8 \\
\hline ZINC01757986 & 294.28 & 0.63 & 102.58 & 9 & 3 \\
\hline
\end{tabular}

$($ ClogP $=$ Calculated partition coefficient; Mol. Wt. = Molecular weight; $2 D P S A=$ Two dimensional polar surface area; $H B A=$ Hydrogen bond acceptor; and $H B D=$ Hydrogen bond donor) 


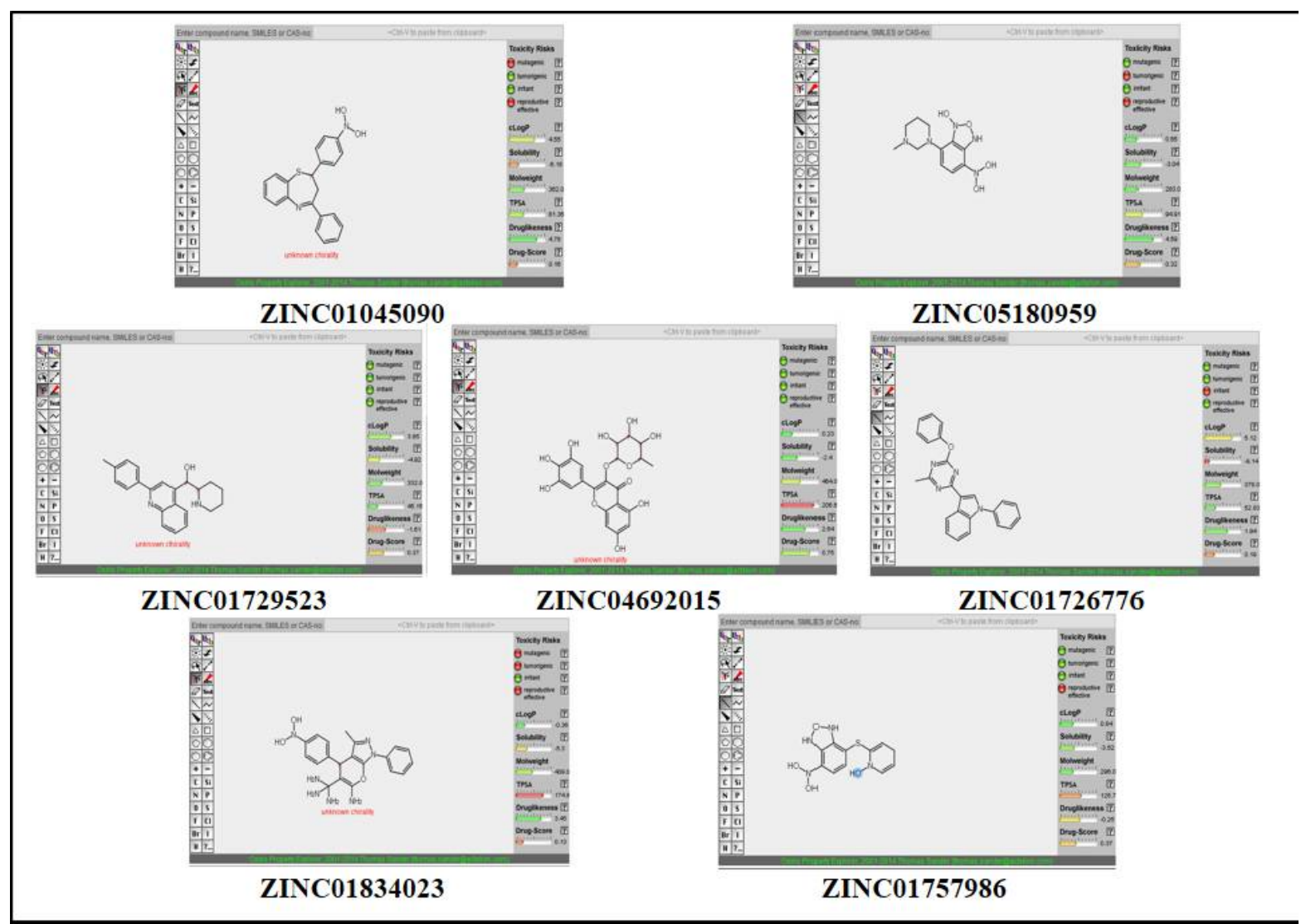

Figure 3: ADME-T profiling and toxicity prediction of the virtually-screened lead compounds [13-15]

\section{DISCUSSION}

The amino acids, TRP250, VAL251, GLY252 and GLU253, are active binding residues present in the binding site for human Myocilin Olfactomedin Domain protein [12]. The identified binding residues involved in the binding of the ligand hexaethylene glycol is used for performing molecular docking simulation studies to identify novel ligand molecule with more binding affinity towards the receptor. Initially the molecular docking simulation process is validated by using re-docking of already bound ligand on the basis of its binding energy and overlay methods, which confirmed the similarity in binding patterns obtained by molecular docking simulation studies with that was occurring inside the human body [11].

After validating the molecular docking simulation technique, the similar parameters were utilised to perform molecular docking simulation based virtual screening by using ligand library " $\mathrm{NCl}$ Diversity Set-Il" containing large number of diverse ligand molecules [13].

The results obtained in this study indicate that Autodock-based virtual screening is very beneficial in screening for the best binding lead molecules from the molecular library containing 1880 diverse ligand molecules on the basis of their binding energies [11]. Two lead molecules ZINC01729523 and ZINC04692015, out of selected top seven lead molecules showed good pharmacokinetic properties, good drug likeness and Drug Score Values. In addition, they had no toxic effects [11,13-15].

\section{CONCLUSION}

Molecular docking simulation-based in silico virtual screening using Autodock is very useful in short-listing potential lead compounds. Two compounds, viz, ZINC01729523 and ZINC04692015, show promising results with potent inhibition of myocilin, good pharmacokinetic properties and absence of toxic effects. These molecules can serve as promising lead compounds for further structure-based discovery of novel drugs for the treatment of glaucoma.

\section{DECLARATIONS}

\section{Acknowledgement}

The authors would like to express their gratitude to all their colleagues and support staff of the laboratory for sharing their support during the course of this research. Immense appreciation 
also goes to all those who directly or indirectly helped in the completion of this research.

\section{Conflict of Interest}

No conflict of interest associated with this work.

\section{Contribution of Authors}

The authors declare that this work was done by the authors named in this article and all liabilities pertaining to claims relating to the content of this article will be borne by them.

\section{Open Access}

This is an Open Access article that uses a funding model which does not charge readers or their institutions for access and distributed under the terms of the Creative Commons Attribution License (http://creativecommons.org/licenses/by/ 4.0) and the Budapest Open Access Initiative (http://www.budapestopenaccessinitiative.org/rea d), which permit unrestricted use, distribution, and reproduction in any medium, provided the original work is properly credited.

\section{REFERENCES}

1. Rao VP, Epstein DL. Rho GTPase/Rho kinase inhibition as a novel target for the treatment of glaucoma. Bio Drugs 2007; 21(3): 167-177.

2. Wang SK, Chang RT. An emerging treatment option for glaucoma: Rho kinase inhibitors. Clin Ophthalmol 2014, 8: 883-890.

3. Pattabiraman PP, Rao PV. Mechanistic basis of Rho GTPase-induced extracellular matrix synthesis in trabecular meshwork cells. Am J Physiol Cell Physiol 2010; 298(3): C749-C763.

4. Goel M. Picciani RG. Lee RK. Bhattacharya SK. Aqueous Humor Dynamics: A Review. Open Ophthalmol J2010; 4: 52-59.
5. Clark AF, Steely HT, Dickerson JE, English-Wright S, Stropki K, McCartney MD, Jacobson N, Shepard AR, Clark JI, Matsushima H, Peskind ER, Leverenz JB, Wilkinson CW, Swiderski RE, Fingert JH, Sheffield VC, Stone EM. Glucocorticoid induction of the glaucoma gene MYOC in human and monkey trabecular meshwork cells and tissues. Invest Ophthalmol Vis Sci 2001; 42(8): 1769-1780.

6. Kanagavalli J, Pandaranayaka PJ, Krishnadas SR, Krishnaswamy $S$, Sundaresan $P$. In vitro and in vivo study on the secretion of the Gly367Arg mutant myocilin protein. Mol Vis 2007; 13: 1161-1168.

7. OhImann A, Tamm ER. The role of myocilin in the pathogenesis of primary open-angle glaucoma. Ophthalmologe 2002; 99(9): 672-677.

8. Vision 2020: The Right to Sight. Global initiative for the elimination of avoidable blindness. Action Plan 20062011, World Health Organisation.

9. Leonord R. Statistics on Vision Impairment: A Resource Manual. April 2002, 5th Edition.

10. Solomon A. Magnitude and Causes of Childhood Blindness and Severe Visual Impairment in Sekoru District of Jimma Zone, South West Ethiopia: The Key Informant Method. Project Report, London School of Hygiene \& Tropical Medicine. 2008-09.

11. Forli S. Raccoon Autodock Preparation Tool. The Scripps Research Institute; 2009.

12. Berman HM, Westbrook J, Feng Z, Gilliland G, Bhat TN, Weissig H, Shindyalov IN, Bourne PE. The Protein Data Bank. Nucleic Acids Res 2000; 28: 235-242.

13. Mujwar S, Pardasani KR. Prediction of Riboswitch as a Potential Drug Target for Infectious Diseases: An Insilico Case Study of Anthrax. J Med Imag Heal Inform 2015; 5(1): 1-10.

14. Lipinski CA. Lead-and drug-like compounds: the rule-offive revolution. Drug Disco Today: Technol 2004; 1(4): 337-341.

15. Sander T, Freyss J, von Korff M, Reich JR and Rufener C. OSIRIS, an entirely in-house developed drug discovery informatics system. J Chem Info Mod 2009; 49(2): 232-246. 